\title{
Commentary: Echocardiography for ischemic mitral regurgitation: It is time to advance the imaging standards
}

Serguei Melnitchouk, MD, and Gus J. Vlahakes, MD

From the Department of Surgery, Harvard Medical School, Boston, Mass; and the Division of Cardiac Surgery, Massachusetts General Hospital, Boston, Mass.

Disclosures: Authors have nothing to disclose with regard to commercial support.

Received for publication Sept 22, 2018; accepted for publication Sept 24, 2018; available ahead of print Oct 30, 2018.

Address for reprints: Gus J. Vlahakes, MD, Division of Cardiac Surgery, Massachusetts General Hospital, 55 Fruit St, COX 630, Boston, MA 02114-2696 (E-mail: vlahakes.gus@mgh.harvard.edu).

J Thorac Cardiovasc Surg 2019;157:1804-5

$0022-5223 / \$ 36.00$

Copyright (c) 2018 by The American Association for Thoracic Surgery

https://doi.org/10.1016/j.jtcvs.2018.09.085

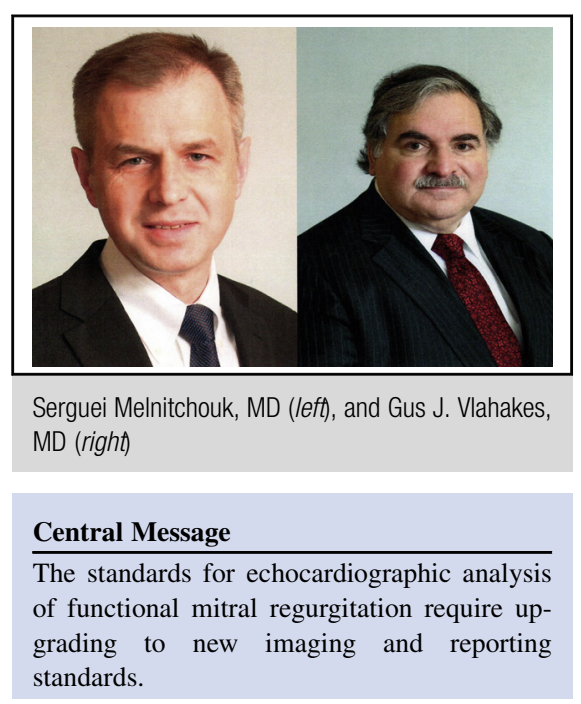

See Article page 1795.
The article by Sun and colleagues ${ }^{1}$ in this issue of the Journal contains a "second message" for those surgeons who treat valvular and ischemic heart disease and for those cardiologists who evaluate these patients. These patients received very sophisticated echocardiographic analysis of their mitral valve anatomy and physiology. Furthermore, this information was gathered in awake patients, and thus under normal loading conditions. The type of echocardiographic assessment needed for ischemic mitral regurgitation is different from that for degenerative disease, in that loading conditions, preload, and afterload can all affect the findings in functional mitral regurgitation. Although it might seem attractive to make all the necessary assessments and decision making at the time of surgery with transesophageal echocardiographic imaging, the aforementioned factors are altered during anesthesia-not only preload and afterload, but also the amount of sympathetic tone present.

Accordingly, surgeons treating valvular heart disease must ensure that reliable echocardiographic imaging and analysis is done before a patient comes to surgery for ischemic mitral regurgitation, and under conditions of optimized medical therapy. In the current state of echocardiography in the United States, the clinical application of echocardiography and the degree of detail that is analyzed and recorded in an echocardiographic report are not adequate to do the type of decision making that is used in this publication. The current standard for a "complete transthoracic echocardiogram" can be found online (https:/iaconlineaccreditation.org/webdriver/Standards/echo/ standards/html/2014/Adult/fs.htm).

To facilitate the analysis and reporting that is needed, many echocardiography equipment vendors offer a valvular heart disease imaging option, such as the TomTec system (Koninklijke Philips NV, Amsterdam, The Netherlands) used by Sun and colleagues ${ }^{1}$ in this study. Although this adds time and complexity to the recording and analysis of images, it is a requirement for optimum management of ischemic mitral regurgitation, as demonstrated by this publication.

Making a substantial change in policy and introducing a new echocardiographic procedure is not a trivial matter. It will need not only engagement the American Society for Echocardiography, the American College of Cardiology, and the American Heart Association, but also significant surgical input from The American Association for Thoracic Surgery and the Society of Thoracic Surgeons. One of the collateral issues that will need to be addressed is reimbursement for a more advanced type of echocardiography, one that takes at least twice the time to perform and analyze as a standard comprehensive transthoracic echocardiogram; in addition, the imaging skills needed by sonographers will need to be upgraded. This is a good opportunity to create a procedure definition for advanced echocardiographic analysis of valvular heart disease, along with appropriate adjustments to reimbursement because of the time and additional skill needed.

In the meantime, the treatment strategy for ischemic mitral regurgitation continues to evolve. Patients with ischemic mitral regurgitation are a complex and heterogenous group, with varying degrees of ischemia, regional wall motion abnormalities, time since infarct, infarct size, and remaining myocardial viability. Despite recent multicenter trials, the question of which patients are best served with mitral valve replacement, ring annuloplasty, 
mechanical support strategy, or optimal medical therapy has not been answered completely. Perhaps more advanced echocardiographic analysis, coupled with further integrative assessment, is the next step in the right direction.

\section{Reference}

1. Sun X, Jiang Y, Huang G, Huang J, Shi M, Pang L, et al. Three-dimensional mitra valve structure in predicting moderate ischemic mitral regurgitation improvement after coronary artery bypass grafting. J Thorac Cardiovasc Surg. 2019;157: 1795-803.e2 